Service social

\title{
Violence conjugale : Étude qualitative sur les comportements d'aide des médecins et des infirmières
}

\section{Geneviève Martin et Francine Lavoie}

Volume 39, numéro 2, 1990

Les problèmes sociaux

URI : https://id.erudit.org/iderudit/706485ar

DOI : https://doi.org/10.7202/706485ar

Aller au sommaire du numéro

Éditeur(s)

École de service social de l'Université Laval

ISSN

1708-1734 (numérique)

Découvrir la revue

Citer cet article

Martin, G. \& Lavoie, F. (1990). Violence conjugale : Étude qualitative sur les comportements d'aide des médecins et des infirmières. Service social, 39(2), 188-216. https://doi.org/10.7202/706485ar
Résumé de l'article

Cet article s'inscrit dans une perspective de prévention secondaire en matière de violence conjugale. Il présente une partie des résultats d'une recherche qualitative menée auprès de vingt sujets, médecins et infirmières, en milieu hospitalier et en pratique privée. Dix comportements d'aide sont regroupés autour de deux axes : l'action directe auprès des femmes violentées et l'action avec les ressources. La discussion porte sur la relation entre intervenants, intervenantes et femmes violentées, sur l'éthique reliée à la suggestion de départ du foyer et enfin, sur la contribution de ces intervenants et intervenantes à l'ensemble du système d'aide en faveur des femmes violentées. 
Geneviève Martin, professeure à l'École de service social de l'Université Laval.

Francine Lavoie, professeure à l'École de psychologie de I'Université Laval.

\section{Violence conjugale Étude qualitative sur les comportements $\mathrm{d}^{\prime}$ aide des médecins et des infirmières ${ }^{1}$}

Geneviève Martin

Francine Lavoie

La violence constitue un problème social sur lequel un débat collectif s'engage de façon plus intense au début de cette décennie. II nous paraît approprié de contribuer à ce débat à l'aide d'analyses résultant d'une démarche de recherche sur la violence faite aux femmes en milieu conjugal. La recherche dont il sera ici question a été menée auprès de vingt intervenants et intervenantes du monde médical, médecins et infirmières appelés à rencontrer les femmes violentées dès les premières heures suivant l'agression ou lors de consultations ultérieures.

Voici la principale question de recherche à laquelle nous apportons des éléments de réponse : quelles sont les réactions des intervenants et intervenantes du monde médical ${ }^{2}$ face aux femmes ayant vécu de la violence conjugale ? Nous cherchons ici à savoir quels sont les types d'intervention envisagés. D'autres écrits ont porté sur les attitudes (Le Bossé, Lavoie et Martin, 1989a) et sur l'évaluation (Le Bossé, Lavoie et Martin, 1989b).

La pratique en milieu médical a été souvent critiquée au sujet de la non-identification de la violence conjugale (Stark, Flitcraft et Frazier, 1979; Commission d'enquête sur les services de santé et les services sociaux, 1987). L'évaluation de la situation de la femme violentée ne révèle pas l'origine réelle du problème tandis que la méconnaissance de la problématique de la violence conjugale semble avoir un impact sur la relation femme violentée-médecin (Bilodeau, 1986). Une étude pancanadienne relate des progrès réalisés dans cette pratique en mentionnant le rôle actif joué par les médecins et infirmières dans les services d'urgence des hôpitaux concernant le 
diagnostic, la prévention et les soins à prodiguer aux femmes violentées (MacLeod, 1987).

Retraçons dans quelques études des façons utiles de traiter la violence conjugale en milieu médical. L'approche la plus susceptible de réussir, une fois la violence soupçonnée, serait celle d'un questionnement direct et sensible à la victime tel que stipulé dans une étude auprès d'une clientèle d'un hôpital général à partir des informations contenues dans les dossiers médicaux et les notes du département de service social (Christiano et al., 1986).

L'hospitalisation est considérée aussi comme un service à offrir aux femmes violentées et parfois recommandé pour assurer la sécurité physique de la femme violentée, pour souligner la gravité de la situation aux yeux de la femme violentée et de son conjoint (Ghent, DaSylva et Farren, 1985) et pour des raisons sociomédicales (Brismar, Bergman, Larsson et Stranberg, 1987).

L'intervention par la prescription de médication est aussi rapportée dans les études, mais ce type d'aide ne semble pas beaucoup préconisé. On souligne le danger de ne faire alors reposer le problème que sur la femme. On croit aussi que les tranquillisants ne vont traiter que les symptômes. Enfin, il serait contre-indiqué de prescrire pour plus de deux semaines à la fois (Ghent, DaSylva et Farren, 1985). La prescription d'analgésiques a été l'aide la plus fréquemment demandée par des femmes violentées appelées à identifier les services requis dans un service $d^{\prime}$ urgence (Goldberg et Tomlanovich, 1984).

Parmi les autres services requis, on note dans cette même étude, la demande de la part de $20 \%$ des femmes violentées pour du counseling pour elles-mêmes et pour de l'aide centrée sur la relation de violence (Goldberg et Tomlanovich, 1984).

Un bon nombre de comportements d'aide en milieu médical sont répertoriés dans quelques recherches : laisser la femme violentée s'exprimer, ne pas décider pour elle, reconnaître son ambivalence, identifier ses forces, lui offrir un soutien, voir à sa sécurité, identifier des scénarios menant à la violence, la référer, bien décrire les services auxquels on réfère, répondre immédiatement sans remettre le rendez-vous, l'interroger directement sur la violence, l'aider à faire un lien entre cet événement et l'histoire passée de sa vie de couple, désapprouver la violence non les personnes, l'informer sur ses droits et sur les ressources, apporter du support à la femme qui décide de passer à l'action, aider à développer des comportements de santé ou à éliminer les problèmes reliés au stress de la violence, l'amener à éliminer son sentiment de culpabilité, l'aider à reprendre contrôle, contrer les mythes qu'elle rapporte, l'aider à se préparer à une 
prochaine crise, tenir des dossiers explicites, assurer un suivi (Lavoie et Martin, 1989).

Peu d'attention ayant été apportée à la systématisation de la démarche d'intervention en milieu médical dans des recherches empiriques sur la violence conjugale, nous croyons que nos résultats de recherche peuvent contribuer à élaborer un modèle de pratique spécifique au monde médical, c'est-à-dire un type d'intervention applicable dans le contexte de la pratique en milieu de santé. Nous aborderons aussi le contenu axiologique d'un modèle, c'est-à-dire l'éthique concernant les justifications des décisions et des actions (Lalonde, 1976) des intervenants et intervenantes du monde médical.

Enfin, compte tenu des travaux recensés, nous retenons, pour notre analyse du thème "Intervention", les dimensions suivantes : 1. L'action auprès des femmes violentées : cette action comprendra, outre les soins physiques, une aide de type support, une aide pour contrer la violence conjugale et une prise de position sur ce problème social; 2 . L'action en relation avec les systèmes environnants : la transmission de l'information sur les ressources et la collaboration avec ces dernières seront déterminantes d'une aide efficace auprès des femmes violentées.

\section{Méthodologie}

\section{Participants et participantes}

L'emploi de la méthode qualitative pour étudier la violence conjugale commence à augmenter et peut permettre d'enrichir l'analyse du phénomène (Knight et Hatty, 1987). Bien que ces auteures valorisent surtout le recours aux témoignages des femmes violentées elles-mêmes, nous soutenons que le discours des intervenants et intervenantes de première ligne est très révélateur et que leur participation à la recherche peut être une occasion de prise de conscience, étape importante dans la recherche sociale (Fine, 1988).

L'échantillon a été constitué de vingt praticiens et praticiennes du monde médical : cinq infirmières et quinze médecins dont neuf hommes et six femmes, selon les critères initiaux (Lincoln et Guba, 1985) de lieux de travail et de sexe. En effet nous comptions interviewer des intervenants de divers lieux de pratique où les femmes violentées sont susceptibles de se présenter : les cliniques d'urgence des hôpitaux (quatre infirmières, quatre médecins), les cabinets privés (huit médecins), les cliniques externes ou de médecine familiale (trois médecins et une infirmière). Il est à noter que la plupart des médecins connaissent plusieurs lieux de pratique jume- 
lant souvent une pratique privée à une pratique à l'urgence ou à la clinique externe.

Nous envisagions d'interviewer un nombre égal d'hommes et de femmes et désirions utiliser la méthode " boule de neige " (Lincoln et Guba, 1985) pour les recruter. La majorité des personnes interviewées ont entre trente et quarante ans (voir tableau 1).

TABLEAU 1

Nombre de sujets par lieu de pratique

\begin{tabular}{|cc|cc|cc|}
\hline Clinique d'urgence & \multicolumn{2}{|c|}{$\begin{array}{c}\text { Unité de clinique } \\
\text { externe }\end{array}$} & \multicolumn{2}{c|}{ Pratique privée } \\
\hline $\begin{array}{ccc}\text { Infirmières } \\
4\end{array}$ & 4 & 1 & 3 & - & 8 \\
\hline
\end{tabular}

Nombre de sujets par âge

\begin{tabular}{|l|ccc|ccccc|}
\hline & \multicolumn{3}{|c|}{ Hommes } & \multicolumn{5}{c|}{ Femmes } \\
\cline { 2 - 8 } & $\mathbf{3 0}$ & $\mathbf{3 0 - 3 9}$ & $\mathbf{4 0}$ & $\mathbf{3 0}$ & $\mathbf{3 0 - 3 9}$ & $\mathbf{4 0}$ & $\begin{array}{c}\text { Incon- } \\
\text { nu }\end{array}$ \\
\hline $\begin{array}{l}\text { Médecins...... } \\
\text { Infirmières..... }\end{array}$ & - & - & - & - & - & 4 & 1 & 1 \\
\hline
\end{tabular}

\section{Procédure}

L'analyse qualitative sera basée sur le contenu manifeste des entrevues. À partir de la recension des écrits et de quelques entrevues préliminaires dans le milieu médical, nous avons élaboré un premier guide d'entrevue soumis d'abord à un processus de révision par les membres de l'équipe de recherche et ensuite à un prétest auprès d'un médecin. Six grands thèmes ont été inclus dans ce guide : le profil de nos sujets, le contexte professionnel, les attitudes des intervenants et intervenantes, l'identification ou le dépistage, l'évaluation et, finalement, l'intervention. Nous cherchions à mettre en évidence les propriétés des données recueillies d'où le caractère inductif de la démarche.

Le type d'entrevue retenu a été l'entrevue semi-structurée, d'une durée d'environ une heure et menée sur le lieu de travail du sujet. L'intervieweur ou l'intervieweuse a joui d'une certaine latitude quant 
à l'utilisation du guide d'entrevue. Ainsi l'ordre et le choix des questions ont pu varier. Notons que des modifications ont été introduites au guide d'entrevue au fur et à mesure du déroulement de la recherche, le principe de saturation des données ayant été adopté (Patton, 1980).

Pour permettre l'obtention de résultats de qualité, c'est-à-dire présentant une certaine fidélité et validité (Lincoln et Guba, 1985), nous avons retenu certaines stratégies dont l'enregistrement et la transcription intégrale des entrevues. En outre, quatre personnes ont participé à la réalisation, à la codification et à l'analyse des entrevues. Le travail d'équipe comporte de nombreux avantages dont une variété de perspectives et d'expertises, la comparaison et la vérification mutuelle des résultats (Lincoln et Guba, 1985). Pour assurer aussi la qualité des données recueillies, les intervieweurs et intervieweuses ont reçu une formation particulière portant sur les techniques d'entrevue.

\section{Mode d'analyse des données}

Pour analyser les données recueillies, nous nous sommes inspirées de Fortin (1988) et avons adapté ce qu'il suggère comme processus séquentiel :

1. La réduction et l'organisation des données.

Cette phase consiste à repérer dans le discours des sujets les éléments les plus significatifs par rapport à chacun des thèmes et sous-thèmes, à les synthétiser, à les illustrer par des citations et à les commenter. Ce contenu a été organisé sous forme de fiche synthétique et correspond à l'analyse intra-sujet/intra-thématique, $c^{\prime}$ est-à-dire l'analyse sur la façon dont chaque sujet s'exprime sur chaque thème.

2. L'interprétation des données.

À l'aide des données ainsi réduites, il devint possible de faire l'analyse trans-sujet/intra-thématique amenant à dégager le patron thématique, c'est-à-dire le procédé implicite chez l'ensemble de nos sujets en regard du thème étudié. L'étude de la cooccurrence associative (Bardin, 1980) a également inspiré notre démarche.

\section{Limites}

Soulignons d'abord que, considérant le petit nombre de médecins et d'infirmières interviewés, nous n'avons pas séparé de façon systématique leurs propos lors de l'élaboration de tous les patrons thématiques. L'objectif a été de jeter un éclairage sur cette pratique 
en milieu médical et de dégager les perspectives de l'ensemble de ce milieu sur telle ou telle position (Patton, 1980).

Quant aux lieux de pratique, tel qu'indiqué précédemment, ils sont fréquentés souvent par le même médecin, tandis que les infirmières ne sont affectées généralement qu'à un seul lieu. Nous noterons des différences de contexte pouvant avoir un impact sur la pratique mais nous n'en avons pas fait une étude comparative exhaustive, ce qui aurait pu enrichir une étude de type qualitatif.

Une autre limite tient au fait qu'il a été difficile d'assurer la caractéristique d'exclusivité de certaines catégories (Bardin, 1980). Des énoncés peuvent parfois être codifiés dans plus d'une catégorie. Ainsi, il fut parfois difficile de distinguer des indicateurs d'identification et d'évaluation, des indicateurs d'attitudes devant l'intervention et l'intervention proprement dite. Nous souscrivons, pour ce type de recherche, à l'idée de souplesse du caractère d'exclusivité d'une catégorie (L'Écuyer, 1984).

Enfin, étant donné le caractère exploratoire du volet qualitatif et la taille de l'échantillon, nous ne pouvons tirer de conclusions définitives ni généraliser à l'ensemble des intervenants et intervenantes du milieu de la santé.

\section{Résultats}

Ce texte décrit les comportements d'aide ou l'intervention subséquente à l'identification (dépistage) et à l'évaluation de la violence conjugale. $S^{\prime} i l$ y a parfois référence à ces autres thèmes, $c^{\prime}$ est qu'on peut retrouver, en milieu médical, le caractère simultané de la séquence identification-évaluation-intervention en matière de violence conjugale. Dix comportements d'aide seront regroupés autour de deux axes principaux : action auprès des femmes violentées et action avec le milieu (voir tableau 2).

\section{Premier axe : action auprès des femmes violentées}

Nous entendons par action l'ensemble des comportements d'aide visant à apporter un changement à la situation en intervenant directement auprès de la femme violentée (Johnson, 1983).

Procurer les soins médicaux. À l'arrivée de la femme violentée, nous avons repéré quatre façons d'agir :

1. Les intervenants et intervenantes peuvent traiter les blessures prioritairement :

" $\grave{A}$ l'urgence, on se limite aux problèmes pour lesquels on est consulté, parce qu'on n'est jamais le médecin traitant, alors elles 


\section{TABLEAU 2}

\section{Intervention : dix comportements d'aide}

1. Action auprès des femmes violentées

- Procurer les soins médicaux

- Offrir un support

- Assurer un suivi

- Utiliser un mode de thérapie

- Divulguer sa position contre la violence

- Contribuer au scénario de protection

2. Action avec le milieu

- Informer

- Référer

- Collaborer

- Intervenir sur les aspects légaux

viennent nous voir pour un problème en particulier qui semble urgent, on détermine son urgence et on le traite, s'il n'est pas urgent, on la réfère au médecin de famille. "

\section{À l'unité de clinique externe :}

«... si tu as cinquante patients qui attendent dans la salle d'attente, tu peux choisir de geler la plaie, de la réparer et de retourner la personne et ça nous arrive tous de ne pas avoir le temps d'en faire plus que ça."

\section{Au bureau privé :}

" elle désirait se confier, se faire traiter et rassurer, s'assurer que ses blessures ne sont pas importantes, elle voulait savoir si elle avait une fracture ou simplement des contusions. »

2. Les intervenants et intervenantes peuvent, à I'occasion du traitement, aborder d'autres aspects du problème et évaluer la situation :

«... j'utilise toujours le temps où je suis toute seule avec la patiente en train de réparer sa plaie. On a toujours cinq, dix minutes pour geler ça, désinfecter, faire les points de suture. $C^{\prime}$ est un excellent moment pour discuter, et les fois que je l'ai fait, moi, ça a été payant dans le sens que j'ai pu dire à la personne que pour ce qui est de sa plaie, je pensais que ça allait guérir, mais que je considérais qu'il y avait un autre problème, et celui-là on peut aussi s'en occuper. » 
À l'urgence :

"Les soins physiques peuvent s'accompagner d'une évaluation de la protection dans l'immédiat; pour le reste de l'évaluation, elle est confiée au service social, à l'enquête de police. »

3. Les intervenants et intervenantes peuvent hospitaliser la femme violentée :

" ... finalement, devant son état de fatigue très important, je l'ai retirée de son milieu familial, je l'ai hospitalisée une semaine et pendant cette semaine-là, on a travaillé beaucoup et elle a finalement admis qu'il y avait de la violence, je l'ai mise en contact avec des ressources sociales et finalement, elle est partie en maison d'hébergement en sortant de l'hôpital. Mais ça a été difficile, tout le long... à éliminer ce problème-là... elle n'avait rien de physique, elle avait un état de découragement et de dépression qui était quand même assez marqué. "

" je l'ai hospitalisée, "je te garde pour te protéger ici, on peut faire autre chose" ${ }^{\prime \prime}, c^{\prime}$ est ça, je pense que si on lui offre une aide concrète, il y a plus de chance qu'elle s'ouvre. "

II n'y a pas cependant unanimité chez nos sujets par rapport à I'hospitalisation :

" je peux la retirer, je peux l'abriter pour la nuit ou pour une période, je peux la référer vers une ressource extérieure, rarement on pourra l'hospitaliser, si c'est simple comme lésion physique ou psychologique, moi, je ne recommanderais pas qu'on l'hospitalise en psychiatrie chez nous, je ne pense pas que ce soit une bonne idée pour la plupart du monde, c'est la dernière chose à faire. "

4. II peut y avoir prescription de médicaments. Voici les circonstances où il y a médication : douleurs vives suite aux coups, insomnie, réactions dépressives, état de crise et de panique, état de stress, absence de fonctionnalité quotidienne, tentative possible de suicide, augmentation de la fréquence des scènes de ménage.

Les types de médicaments suggérés sont : les sédatifs légers, les psychotropes pour diminuer l'anxiété et favoriser le sommeil, et les antidépresseurs.

Les mises en garde rapportées par le médecin sont les suivantes : ne jamais prescrire plusieurs médicaments à la fois, indiquer à la femme violentée le danger d'accoutumance, assurer un suivi de cette médication et la cesser dès que la femme est mieux.

Les raisons de ne pas prescrire :

"... ça peut être plus dangereux..."

«... c'est le temps qu'elle vive le problème et qu'elle réagisse, il ne

faut pas l'endormir... moi je n'ai pas tendance à endormir. " 
La tendance générale est de ne pas utiliser de médicaments pour traiter le problème de violence comme tel. Tous s'entendent pour ne pas voir la médication comme la solution idéale. Cependant, la gravité des réactions connexes (telle la dépression) ainsi que la nécessité pour la femme d'être en état de prendre des décisions importantes (ne pas faire d'insomnie) amènent la plupart des médecins à prescrire des médicaments en petite dose :

" c'est une arme à deux tranchants, ça peut aider à perpétuer le problème."

Offrir un support. Par delà les soins médicaux, nous pouvons retracer des interventions de type support.

Un médecin insiste aussi sur le support à la patiente pour qu'elle requière l'aide de son milieu familial :

«... supporter à aller chercher de l'aide ailleurs dans le milieu familial en ayant identifié bien sûr les forces, les faiblesses du milieu familial. »

Parmi les attitudes reliées au support, nous retraçons: la préoccupation d'établir une relation de confiance, le respect du rythme de la cliente et l'offre de sa disponibilité. De plus, un sujet insiste sur le désir d'engager la cliente dans la solution de son problème :

" je pense que le principal problème, c'est de ne pas faire la job à la place de la patiente, il faut supporter, mais il faut à mon avis, compte tenu des capacités de la patiente, la supporter dans l'acquisition de sa liberté. »

Quelques techniques sont utilisées pour manifester ces attitudes, à savoir :

l'écoute active :

«... c'est vraiment plus une oreille réceptive, voir si elle veut en parler... je vais certainement lui poser quelques questions pour essayer de favoriser qu'elle en parle, et essayer d'établir le pattern. »

la ventilation :

" c'est au moins être capable de venir vider leur sac et de se révolter dans un espace clos et à quelques reprises."

la validation de l'expérience :

" ça va les aider à poursuivre leurs démarches et à se faire confirmer qu'elles ont des droits et qu'elles ne sont pas folles."

Les sujets utilisent aussi la déculpabilisation, la réassurance et I'universalisation (technique permettant d'apprivoiser des émotions difficiles à supporter en sachant qu'elles sont communes à tout être humain dans des circonstances semblables) : 
"... être déculpabilisante aussi et leur dire : "ce n'est pas forcément mérité ce qu'elles ont eu", qu'on en a déjà vu, qu'elles ne sont pas les seules, qu'on sait comment intervenir et qu'on va assurer un suivi."

Les contraintes reliées à ce soutien relèvent du manque de temps et d'énergie :

" c'est difficile de traiter ces patientes-là, ce sont des gens qui prennent du temps, il faut que tu donnes de l'énergie, tu es fatigué."

" on peut l'aider d'un point de vue physique, on peut essayer... psychologique aussi mais on n'a pas beaucoup de temps, on fait ce qu'on peut... le support si tu veux."

Certains se sentent démunis :

"Au départ, on est démuni... l'écoute... oui je suis bien d'accord (avec ça) mais on ne va pas loin avec ça, ça fait du bien aux gens et je leur dis: "pleurez, pleurez votre saoul, si vous voulez ici, c'est parfait ça, ça va vous faire du bien, pour vous donner un peu d'énergie pour affronter", et après ça l'intervention c'est quoi ? Pas plus que ça."

Un médecin relie l'intervention de type support au peu de pouvoir du médecin :

«... il y en a une qui est venue me voir à quelques reprises pour me parler de ses problèmes, elle venait surtout pour un support moral, mais je n'ai pas plus de pouvoir que ça. Les médecins n'ont pas plus de pouvoir que ça dans cette intervention-là, dans ce genre de problèmes-là, le seul pouvoir que j'ai c'est le pouvoir de constatation, c'est tout. »

Assurer un suivi. Quelques propos de médecins et infirmières nous ont illustré comment le support aux femmes violentées pouvait se poursuivre, c'est-à-dire comment ils et elles envisagent le suivi à l'aide d'autres rencontres avec les femmes violentées. Utilisant la cooccurrence associative (Bardin, 1980), nous pouvons apporter des appuis et des oppositions de nos sujets à faire un suivi avec les femmes violentées (voir tableau 3).

Les appuis peuvent exprimer une tendance à voir le suivi comme une fonction s'apparentant au " follow-up » ou au contrôle médical : on veut mesurer l'évolution du problème de violence et le degré de dangerosité, on veut référer à des spécialistes si le problème demeure, de plus on désire accompagner, être disponible et sécuriser. Un médecin fait part de la compréhension psychosomatique, c'est-à-dire des effets sur le physique causés par le problème de la violence vécue. 


\section{TABLEAU 3}

\section{Des appuis et des oppositions à faire un suivi Quelques propos des médecins et infirmières}

Pour faire prendre cons-
cience du lien entre les
problèmes de santé et la
violence

Pour s'occuper de gens qui sont très seuls

Pour voir le cheminement

Pour aider par rendezvous de contrôle si la femme violentée dissimule le fait d'être battue

Pour devenir plus directif

\section{Appuis}

« moi je sème des graines, je ne sais pas ce qui va pousser, je ne peux pas vivre à la place des autres, mais comme médecin j'ai une crédibilité et une expertise pour dire à quelqu'un qu'il y a un lien... je prends position sur les liens entre leurs malaises et la situation... et j'offre ma disponibilité pour en rediscuter si ça les intéresse, et d'habitude, je m'arrête là, la fois d'après c'est eux autres qui me relancent sur le sujet en général. »

«... c'est un suivi qui est assez serré dans le sens que c'est pas une fois par mois, $c^{\prime}$ est une fois par semaine ou une fois aux 15 jours, même c'est pas nécessairement 1 heure ou 30 minutes, au moins quelques minutes, parce que ce sont des gens que je perçois comme étant très seuls, même les meilleurs amis ne peuvent pas s'en occuper. "

« ... il faut voir comment ça évolue, est-ce que ça évolue dans le bon sens?»

"Je ne pense pas qu'on peut obliger quelqu'un à l'admettre, moi je pense que ces femmes-là, je les revois en contrôle, si elles acceptent, elles ne reviendront peutêtre pas, mais je leur donne un rendezvous de contrôle pour réévaluer leur état physique, supposément après... c'est comme ça que j'ai fait pendant les grossesses, ça s'est fait petit à petit, c'est pas fréquent qu'elles le disent d'emblée. "

«... et de dire à la patiente : Va-t-en de là, tu vas mourir." 


\section{Appuis}

Pour amener à d'autres références

Pour rester disponible après la référence aux gens plus habilités
«Le suivi, ça peut être le point de départ de l'intervention auprès des autres intervenants dans la société. "

" Je ne peux pas dire que je fais beaucoup de suivi, le seul suivi que je fais, c'est que si je la réfère aux gens qui sont plus habilités à s'en occuper et que si la situation se reproduit, et que la dame a besoin encore d'aide, ou de consultation ou quoi que ce soit, je suis toujours disponible, c'est le suivi que je fais."

\section{Oppositions}

Parce que préfère les référer ailleurs et ne pas les revoir

Parce que ne servirait qu'à l'intervenant, pas à la patiente

Parce que n'a pas l'expertise
«... je pense que je ne peux pas en faire beaucoup de suivi, la seule chose que je sais c'est que quand elle revient, je lui pose la question : "C'est y mieux, c'est y réglé ?" "Oui ça va", mais autrement, je peux les diriger ailleurs, souvent chez les psychologues, mais pas les revoir.»

" il faudrait dire qu'un des problèmes c'est que moi je ne fais pas vraiment de suivi, je ne les favoriserais pas non plus dans ma pratique, ça fait que même si j'avais un feed-back, ça serait juste pour mon information, je ne continuerais pas à participer à autre chose, avec ou sans raison, j'ai l'impression d'abandonner. "

" je n'ai aucune expertise et aucune compétence dans ça. Si la dame en question avait voulu être suivie, je lui aurais offert de se faire suivre par quelqu'un d'autre que moi, toujours pour un meilleur service. Personnellement, je ne verrais pas mon utilité là-dedans à moins d'avoir reçu une formation dans ce domaine-là, je pourrais passer à côté du problème. » 


\section{TABLEAU 3 (suite)}

\section{Oppositions}

Parce que le rôle, à l'urgence, c'est de déceler
"Nous on essaie de déceler, ensuite de ça on va référer... le principal point important, moi je trouve, à l'urgence, c'est de s'en apercevoir... si on ne les décèle pas à l'urgence, elles consultent pour des choses et elles s'en retournent après, c'est bien de valeur, mais il n'y aura pas de suivi." "

Parmi les oppositions au suivi, on retrouve des éléments contextuels reliés au rôle de l'urgence concernant le dépistage, des éléments professionnels (manque d'expertise sur le problème de violence conjugale) et des éléments personnels reliés au sentiment d'avoir abandonné une patiente.

Utiliser un mode de thérapie. Nous avons voulu savoir si l'intervenant ou l'intervenante faisait de la thérapie et comment il ou elle la décrivait.

Un médecin cite l'approche psychodynamique :

"La forme de thérapie que j'utilise, c'est I'approche psychodynamique, ça fait deux ans que j'ai commencé ma formation et j'utilise ces thérapies-là, je rencontre les personnes une heure par semaine pendant un temps $\mathrm{X}$ et j'utilise le transfert comme outil de travail. "

La pensée de quelques médecins ayant répondu positivement semble bien représentée dans les propos tenus par l'un d'eux sur la thérapie individuelle :

«... et qu'est-ce qu'elles peuvent faire face à ça... décider de s'en aller, décider de régler le problème en ayant une thérapie, en parlant au conjoint ou alors choisir explicitement de continuer à vivre dans cette situation-là... je leur précise que le fait de ne pas faire de choix leur fait faire automatiquement le choix de subir cette situation-là, qu'elles sont "faites", qu'elles sont coincées. Je veux que tout de suite, elles choisissent une des trois solutions. C'est souvent ça que je fais, c'est malheureusement le type d'ultimatum..."

Un autre médecin précise qu'il n'est pas là pour aider madame seulement et réfère par ses propos à la thérapie avec le système conjugal et à la thérapie familiale :

«... souvent je vais dépasser le stade de l'évaluation pour voir si dans trois ou quatre rencontres quelque chose peut être mobilisé à l'intérieur du système, mais là, à ce moment-là, je ne me décris pas 
comme le thérapeute de madame, c'est un couple que d'emblée je vais voir pour démontrer que je suis entre adultes."

Plus loin ce même médecin indique qu'il tient aussi compte de la présence des enfants.

Plusieurs sujets disent ne pas faire de thérapie parce qu'ils n'en ont pas la formation, n'ont pas le temps, ont besoin de travailler en collaboration avec d'autres ou ont un manque d'intérêt.

Divulguer sa position contre la violence. À la question "Pensezvous qu'un médecin ou une infirmière doit prendre position contre la violence en le verbalisant à la femme lors de la consultation ? ", tous les sujets ont répondu positivement. Le libellé de la question pouvait entraîner cette réaction. II est donc très important de dégager quelques tendances, concernant la divulgation.

Première tendance : Dire qu'on juge la situation inacceptable et en donner les raisons. Celles-ci se rapportent aux droits des individus, à leur valeur comme personne, au contexte religieux et social.

Une infirmière dit à une femme violentée :

"Vous savez vous n'êtes pas obligée d'accepter ça la violence conjugale, c'est pas dans nos mœurs, et ce n'est pas quelque chose dans la société qu'on doit accepter, vous avez le droit de vous défendre contre ce problème-là et vous avez des droits, aussi vous pouvez les faire respecter vos droits."

Un médecin s'adresse ainsi à la femme violentée :

"Écoutez, à moins que vous n'ayez plus le goût de vivre, c'est dangereux ce que vous faites là, il faut que la situation se règle d'une manière ou d'une autre. "

Des médecins insistent sur la valeur de la personne quand ils disent à la femme violentée :

«... "personne ne devrait être battu, ce n'est pas parce que tu n'es pas bonne, ce n'est pas parce que tu n'es pas belle, ce n'est pas parce que tu n'as rien que tu devrais subir ces choses-là, personne ne devrait être exploité dans ces situations, tout le monde a une valeur", essayer de la mettre dans un contexte qu'elle est une personne qui a des droits, qui a des bonnes caractéristiques de personnalité, "tu ne devrais pas être traitée comme ça", parce que souvent les femmes battues, je trouve que ce sont des femmes qui sont dévalorisées beaucoup et elles ne pensent pas grand-chose d'elles-mêmes (...) je pense que ce que tu vis, c'est pas quelque chose qui peut améliorer l'image que tu as de toi-même... ce que tu vis c'est la force de l'agresseur, $c^{\prime}$ est de te faire croire que tu l'as bien mérité et $c^{\prime}$ est pour ça que tu penses qu'il n'y a rien à faire... j'ai beaucoup $d^{\prime}$ intolérance face à ce genre de violence-là. »

"Oui, dans le sens où, quand on ne prend pas position moi je pense qu'on n'aide pas, prendre position pas pour dire aux gens quoi faire, 
se prononcer sur le fait qu'on n'est pas obligé de vivre dans un contexte de violence... on n'est pas, les êtres humains, faits pour endurer la violence, ce n'est pas notre job sur terre... si tu veux ramasser des indulgences, dis-le-moi quand tu en auras assez, une plénière, tu vas être correcte, on pourra passer à d'autres choses... »

Deuxième tendance : Prendre position, c'est aussi manifester sa solidarité et insister pour de la sensibilisation :

«Je pense que la femme doit sentir qu'on est solidaire... Si la relation est bien enclenchée, elles le peuvent, je lui dis que je trouve que c'est une situation qui est inacceptable, qu'elle devrait réagir à ça et que je me sens solidaire d'elle et que je suis prête à l'aider. " "Moi, je pense que oui (prendre position) en tant que femme et en tant qu'infirmière aussi, c'est pour ça que j'aimerais ça qu'il y ait plus de sensibilisation au niveau des infirmières, surtout au niveau de l'urgence, on est quand même susceptible d'en voir plusieurs. "

\section{L'engagement personnel est aussi relié à cette solidarité :}

"... je pense que c'est le rôle de tout individu de prendre position contre la violence et je ne pense pas que ce soit spécifiquement relié à une profession, c'est personnel comme intervention, du fait que nous autres on est en contact avec trop de gens, je pense qu'on ne doit pas négliger d'intervenir là-dessus, moi je prends position... »

Troisième tendance : L'incitation à réagir à sa position contre la violence :

«... j'ai pris sa part dans le sens que j'ai dit carrément que c'était tout à fait inadmissible et qu'il y avait des lois au Québec et qu'elle pouvait porter plainte et que si elle voulait porter plainte je faisais son dossier de façon très explicite avec un dessin, toute l'affaire... je lui ai dit : "je suis prête à aller témoigner sur le fait que j'ai vu ces blessures-là", j'ai l'impression que ça l'a galvanisée un peu parce qu'elle n'avait pas porté plainte et elle est allée porter plainte l'aprèsmidi même." " d'aide :

Quatrième tendance : Cette position se traduit par une offre

" (à la femme violentée) écoute bien, ces choses-là, ça doit pas exister, tu vis très mal et tu sais le pourquoi de ça. J'ai dit il y a quelque chose pour toi... tu l'aimes peut-être ton chum mais... pour le moment c'est pas possible de vivre comme ça, tu le vois ta petite fille s'en ressent aussi... je vais prendre tous les numéros nécessaires, aussitôt que tu m'appelles je fais les demandes pour pouvoir te trouver une petite place où tu seras en sécurité, tu vas manger trois fois par jour, ta petite fille aussi, tu vas pouvoir continuer à aller à l'école, tu vas être en sécurité. "

"C'est évident qu'on doit s'impliquer... bien, je pense que toute personne, c'est pas le médecin, toute personne qui a affaire à la 
violence devrait démontrer que c'est inacceptable dans une société d'user de pouvoir, que ce soit envers les enfants, envers la femme ou envers d'autres personnes, ça peut être des vieillards aussi, il y a beaucoup de vieillards violentés, alors c'est inadmissible que ce soit comme ça, et qu'il y a des mesures qui peuvent être prises et qui doivent être prises; maintenant il reste la liberté de la personne toujours, c'est difficile de se substituer à ça, mais je pense que c'est important de prendre position, oui. »

Force nous est de constater enfin que la prise de position n'est pas automatiquement empreinte d'empathie :

"Oui, je pense qu'il faut que ce soit dit clairement (s'adressant à la femme violentée) : "Je ne comprends pas comment vous pouvez penser que ça peut avoir du bon sens, se laisser taper, trouvez-vous que ça a de l'allure ? La guerre, êtes-vous pour ça ?... Personne ne peut être pour la guerre"

Contribuer au scénario de protection. Dans cette section sur l'action directe auprès des femmes violentées, nous n'aborderons ici que les suggestions sur une mesure de protection portant sur le départ du foyer.

Les suggestions sont vues comme des conseils, des recommandations, des incitations à l'action. C'est la femme violentée qui prend ensuite l'initiative de l'action :

" je leur dis de ne pas retourner (au foyer) de ne pas aller sur les lieux tout de suite, surtout si elles n'ont pas fait des plaintes, de toute façon si elles ont fait une plainte, ça ne change pas grand-chose, si elle retourne là il va être plus enragé, si jamais il apprend qu'elle a fait une plainte, ça c'est leur peur : la menace de se faire faire la même chose encore..."

La suggestion devient parfois une directive. Ainsi un médecin dit à la femme violentée :

" ... écoute, tu sors de là, il faut que tu sortes de là, c'est très malsain pour toi et ton enfant... tu vois bien que tout ce qui se passe c'est parce que t'as un stress énorme sur tes épaules, puis j'ai dit qu'il y avait un moyen d'enlever ce stress-là. "

Concernant le départ du foyer :

"... ça $\mathrm{m}^{\prime}$ est déjà arrivé de suggérer à une madame, de dire : écoutez si vous ne voulez pas rentrer chez vous, nous autres on fait des téléphones, tout ça."

Ce type de suggestions est assorti de conditions et du respect de I'autonomie de la femme violentée :

"... je pense qu'il faut qu'elle mûrisse et qu'il y ait un temps pour quitter et qu'elle décide elle-même, parce que si on prend une décision à sa place, probablement que la femme violentée va $y$ 
retourner avec son mari... si c'est sous l'influence d'une personne qui connaît à peine la femme en question... pourquoi est-ce qu'elle me ferait confiance et qu'elle dise : oui l'infirmière me l'a dit, je l'ai vue une demi-heure et elle $\mathrm{m}^{\prime} \mathrm{a}$ dit de quitter mon mari..."

"... et lui dire, il faut qu'elle fasse une démarche si elle est prête naturellement parce qu'elles ne sont pas toujours prêtes à quitter le foyer. "

\section{Deuxième axe : action avec le milieu}

Action signifie ici l'ensemble des comportements d'aide visant I'utilisation des ressources du milieu en faveur de la femme violentée.

Informer. L'ensemble du scénario de protection pour la femme violentée sera fonction des informations connues sur les ressources.

Parmi les ressources mentionnées par nos sujets pour la protection immédiate, nous trouvons: le service de police, Urgence Sociale, SOS Violence conjugale, InfoSanté. Lorsque I'hébergement est requis, les organismes suivants sont nommés : les Maisons de Femmes, la Maison Kinsmen, la Maison Hélène-Lacroix, la Maison des Femmes immigrantes, le Centre-Femmes du YWCA. Lorsqu'il s'agit de consultation sur les aspects légaux, les policiers et les avocats sont indiqués. Nos sujets savent que des consultations psychosociales peuvent être fournies par des travailleuses et travailleurs sociaux rattachés au centre hospitalier ou au CLSC. Des psychologues et des psychiatres peuvent aussi être recommandés.

Ces informations s'obtiennent soit par un cardex portant sur toutes les ressources sociales disponibles à l'urgence (l'infirmière ou la travailleuse sociale en fait part au médecin), soit par un document portant sur les ressources à la clinique externe. Quant à la pratique privée, les informations relèvent de l'initiative de l'intervenant ou de l'intervenante.

Lors de la transmission des informations, un médecin fait le lien avec la motivation nécessaire au changement :

«... je suis prête à faire beaucoup pour les épauler mais il faut absolument qu'elles prennent conscience de la gravité de leur problème et qu'il y a plusieurs ressources pour s'en sortir mais qu'il faut leur bon vouloir pour ça.»

Interrogé sur les mesures qui devraient être prises dans le milieu de travail pour améliorer l'intervention auprès des femmes violentées, un médecin dit :

"Ce serait au niveau de la documentation, des ressources, être plus au courant de ce côté-là, avoir quelque chose à offrir plus rapidement, c'est surtout ça, avoir l'aide de personnes ressour- 
ces dans un milieu assez proche, savoir où sont les personnes ressources..."

Le manque d'information souligné par un médecin de l'unité de clinique externe conditionne son action au niveau du dépistage :

"... les médecins ne sont pas portés à dépister des problèmes pour lesquels les ressources ne sont pas évidentes. »

Référer. Ce type d'intervention s'exerce habituellement à l'intérieur du centre hospitalier, avec le réseau des affaires sociales ou avec le réseau des ressources alternatives.

La référence peut se faire dès le constat de la violence à l'arrivée :

"Oui, je les appelle (SOS Violence conjugale) ils ont des maisons d'hébergement eux avec toute l'équipe d'intervenants sur place. C'est parce qu'il faut répondre sur le moment, tu ne peux pas attendre au lendemain, tu ne peux pas mettre une situation comme ça sur la glace comme on fait en médecine, on met des situations sur la glace...»

Tenant compte de la répartition des rôles à l'urgence, c'est souvent l'infirmière qui réfère :

«... si les femmes violentées arrivent en soirée et qu'elles doivent sortir de leur milieu, c'est l'équipe, c'est souvent l'infirmière qui va s'occuper de faire les téléphones, trouver une place pour qu'elles aillent coucher là... un hébergement pour la nuit... "

Un médecin souligne l'avantage à ce que ce soit l'infirmière qui fasse alors l'entrevue :

" je pense que ça aide un peu... ça rassure un peu... elle a été violentée par un homme, ça fait qu'elle n'est pas trop intéressée à dialoguer ou échanger avec un homme dans l'immédiat... si ça vient juste d'arriver. "

On réfère aussi pour de l'aide individualisée et de la psychothérapie :

" je réfère souvent à la psychologue, celles qui ont la possibilité de payer... elles vont aller voir la psychologue tout dépendant de la gravité, je les réfère au CLSC ou au psychiatre très rarement. "

La référence peut aussi se faire après un certain travail sur le problème :

" je vais la revoir quelques fois avant de référer, je veux savoir qu'estce qui en est de ça, sinon, et c'est la même chose qu'en spécialité... rencontrer, bien connaître et là, référer. "

" je pense que les services sociaux sont susceptibles, lorsqu'on est rendu au bout de notre rouleau, de nous orienter et de les évaluer. " " moi mon bout de travail c'est de l'amener à croire et à voir un lien entre la violence qu'elle vit et ses problèmes... et ça peut prendre 
des années... quand une personne a besoin d'une aide plus spécifique... si tu veux, se renforcir quasiment pour prendre des décisions éclairées par rapport à quelqu'un qui l'écrase, entreprendre des procédures légales, être hébergée quand la situation devient flambante, je peux référer au réseau public, au CLSC, au réseau privé. »

Il est aussi intéressant de noter que lorsqu'un médecin réfère une femme violentée, il ne la perd pas nécessairement de vue :

«... ça m'arrive de faire affaire avec le CLSC, je vais appeler I'infirmière du CLSC (de la région de la femme violentée), je vais lui demander de faire une évaluation à la maison, s'ils ont besoin d'un travailleur social on pourra en discuter. "

La référence peut concerner le deux conjoints. Les médecins réfèrent soit au CLSC où il se fait de la thérapie conjugale de type systémique, soit au CSS ou à des psychologues en privé.

La référence peut se faire pour seulement un des conjoints :

" ça peut arriver qu'on réfère en psychiatrie, en travail social, qu'on réfère le bonhomme pour un problème sexuel en sexologie par exemple, qu'on le voit même, mais plus souvent c'est plus utile qu'il ait son médecin à lui d'un autre côté, dépendant du cas, ça arrive fréquemment qu'on a à agir. »

Quelques difficultés reliées aux références sont aussi exprimées. La référence au psychologue n'est pas vue comme une solution :

" j'aurais pas eu tendance à référer une femme comme ça à un psychologue, je pense que c'est pas elle le problème, ça peut être une solution partielle."

"Il y a des difficultés reliées au coût des consultations en clinique privée, avec les travailleurs sociaux et les psychologues; quant au Centre de thérapie conjugale, c'est long avant d'avoir un rendezvous."

On souligne aussi quelques difficultés, dont celle d'avoir des ressources en pleine nuit, en situation de crise. De plus, il n'y a pas de protection lors du harcèlement verbal :

" je pense que quand il y a des appels de l'ex-conjoint une fois qu'elle reste dans la résidence et que lui est obligé de partir et qu'il y a des appels, qu'il y a du harcèlement verbal, je pense que cette femme-là devrait avoir au moins un ombudsman parce que les policiers ne s'en mêlent pas nécessairement. "

Des sujets nous ont mentionné la lourdeur du système, le délai entre la référence et le moment où la femme violentée est rencontrée. Il est aussi fait mention du non-intérêt de certains travailleurs sociaux et travailleuses sociales à s'occuper de ces cas-là. Un sujet exprime ainsi les mesures qui semblent les plus utiles : 
" je dirais que c'est premièrement d'identifier les ressources disponibles pour les références... la pertinence d'utiliser une ressource face à une autre et de préciser les mécanismes de référence et de transfert... on pourrait faire ça sur une échelle métropolitaine. Deuxièmement, d'identifier les cas à risque... Troisièmement, de savoir, de bien comprendre la définition de la violence conjugale. »

Collaborer. Notons d'abord qu'entre collègues médecins, la collaboration peut aller de la "disponibilité à écouter " à la " discussion de cas lourds".

Un médecin croit à l'importance du rôle du médecin et à la spécificité des autres intervenants et intervenantes :

« $j$ 'ai besoin de travailler avec d'autres gens et je ne peux pas avoir la formation pour faire ça tout seul, ça ne m'intéresse pas, ça $m^{\prime}$ 'intéresse beaucoup de dépister ces problèmes-là, de les évaluer et d'être le chef d'orchestre... on a une job de pivot là-dedans. "

La collaboration sous forme d'un rapport écrit est appréciée :

«... les psychologues qui ont vu les patientes et qui m'ont écrit... j'ai toujours apprécié mais c'est exceptionnel compte tenu du nombre de références faites chez les psychologues."

La collaboration avec les travailleuses et travailleurs sociaux se fait plus expéditive si on craint la violence faite aux enfants :

"Oui, c'est certain... puis dans ces cas-là, je n'hésite pas, j'appelle toujours la journée même le travailleur social puis là on se pose toujours la question: est-ce qu'on devrait aviser notre DPJ ? Souvent, je ne sais pas, on va faire l'enquête la plus rapide pour être certain que les enfants ne seront pas violentés."

La collaboration avec les policiers paraît positive :

" ... ils (les policiers) acceptent d'aller conduire la dame à ces placeslà (Maisons de femmes), ils font des compromis, ils ne sont pas supposés faire ça, mais quand on est mal pris, on a de bonnes relations avec eux autres, ils font des compromis."

Les références aux maisons d'hébergement n'entraînent pas nécessairement une collaboration par la suite :

«... on en a entendu parler, mais on n'est jamais allé sur les lieux. »

«... je ne sais pas vraiment l'impact que ça peut avoir, je n'ai pas de suivi par la suite."

Intervenir sur les aspects légaux. L'agir, ici, peut être placé sur un continuum où l'on trouve à un extrême la référence aux intervenantes et intervenants psychosociaux, aux avocats et avocates, au service de médiation conjugale et familiale pour des informations à caractère légal et, à l'autre extrême, l'initiative de l'intervenant ou de l'intervenante à appeler la police. À mi-chemin, quelques sujets informent les 
femmes violentées sur leurs droits et sur les nouvelles lois (Gouvernement du Québec, 1985-1986). D'autres insistent pour que la femme porte plainte elle-même.

Quelques positions sous-jacentes sont soulignées. Une infirmière croit qu'il ne fait pas partie de son rôle professionnel d'intervenir sur les aspects légaux; elle le ferait toutefois à titre personnel. Un médecin est réticent à user de son influence pour une démarche aussi personnelle de la part de la femme violentée, tandis qu'un autre hésite à dénoncer s'il n'y a pas de réelles mesures de protection pour la femme violentée. En général, on constate que bien peu d'informations à caractère légal sont transmises. C'est à d'autres ressources du milieu qu'on demande de le faire. Il y a aussi des réticences à appuyer la plainte.

Aucun médecin ni aucune infirmière ne sont allés en cour pour une cause impliquant une femme violentée. Interrogés sur leurs réactions à une demande de témoignage en cour, quelques-uns ont eu des réactions positives, tandis que d'autres ont exprimé des réactions négatives (voir tableau 4). Certaines de ces réactions se sont retrouvées chez un même sujet, ce qui dénote une certaine ambivalence sur la question.

La solidarité féminine semble s'exprimer par la réaction d'une infirmière voulant que ce témoignage en vaille la peine s'il est bénéfique pour au moins une femme.

Deux questions préoccupent les médecins : est-ce qu'à la cour, on va les mettre "en boîte»? Et qu'en est-il alors du secret professionnel ? Par ailleurs, une infirmière s'interroge sur la place de I'infirmière en cour : ne serait-ce pas plutôt la place du médecin ?

II appert donc que ces réactions et questions dénotent le caractère ambigu des responsabilités en matière légale.

\section{Discussion}

La pratique révélée dans ce tableau issu de nos données soulève plusieurs enjeux dont : 1) la nature de la relation de travail à établir avec la femme violentée, 2) l'éthique professionnelle et 3) la contribution de l'intervenant ou intervenante du monde médical au système d'aide en faveur de la femme violentée.

\section{Nature de la relation de travail}

Pour améliorer cette pratique, ce n'est pas l'ajout d'une catégorie pathologique «femme violentée » qui serait le plus utile, mais bien l'examen de la nature de la relation femme violentée-médecin 


\section{TABLEAU 4}

\section{Réactions devant le témoignage en cour relativement à une situation de violence conjugale}

\begin{tabular}{|c|c|}
\hline Réactions positives & Réactions négatives \\
\hline $\begin{array}{l}\text { Le constat des blessures peut } \\
\text { être apporté en preuve. }\end{array}$ & $\begin{array}{l}\text { La pertinence de cette contribu- } \\
\text { tion est mise en doute. }\end{array}$ \\
\hline $\begin{array}{l}\text { Le témoignage est vu comme } \\
\text { partie intégrante du métier. }\end{array}$ & $\begin{array}{l}\text { La confidence au médecin ne } \\
\text { constitue pas une preuve } \\
\text { légale. }\end{array}$ \\
\hline $\begin{array}{l}\text { La préparation au témoignage } \\
\text { est envisagée. }\end{array}$ & $\begin{array}{l}\text { Un bon dossier contenant un } \\
\text { rapport médical peut remplacer } \\
\text { un témoignage. }\end{array}$ \\
\hline $\begin{array}{l}\text { Le témoignage en vaut la peine } \\
\text { s'il est bénéfique pour au moins } \\
\text { une femme. }\end{array}$ & $\begin{array}{l}\text { Ce genre de procès est souvent } \\
\text { remis. }\end{array}$ \\
\hline $\begin{array}{l}\text { Il ferait plaisir à un médecin } \\
\text { d'aller en cour. }\end{array}$ & $\begin{array}{l}\text { En cas de divorce, les règles ne } \\
\text { sont pas claires concernant les } \\
\text { préjudices causés par la } \\
\text { violence. } \\
\text { II y a des craintes et hésitations. } \\
\text { La démarche est vue comme } \\
\text { désagréable, non pertinente, } \\
\text { détestable et perçue comme } \\
\text { une catastrophe. }\end{array}$ \\
\hline
\end{tabular}

(Warshaw, 1989). Cette constatation émane d'une recherche menée à partir de cinquante-deux dossiers de l'urgence d'un hôpital public. Cette étude rapporte que des intervenants et intervenantes écrivent au dossier des histoires sociales a-historiques, dans le sens où l'événement de violence est situé hors du contexte où il a eu lieu, sans indiquer par qui il a été perpétré, comment, etc. $\mathrm{N}^{\prime}$ y a-t-il pas danger alors, selon l'auteure, que la rencontre en milieu médical ne produise un autre type d'abus, où l'effet de l'agression est tenu en compte sans que la personne abusée ne soit supportée. Cette dernière pourrait se sentir doublement opprimée (Stark et Flitcraft, 1988). Vue sous cet angle, la relation de travail peut être difficilement symétrique, c'est-àdire fondée sur l'égalité (Walzlawick, Helmick-Beavin et Jackson, 
1972). Nos données, tout en apportant des aspects positifs, notamment au niveau du support dans les interactions, comprennent peu de traces d'un partage d'objectifs avec la patiente ni de réelle participation à l'orientation du travail. On retrouve peu de rétroaction des femmes violentées aux suggestions des intervenants et intervenantes. Cosgwell (1987), dans une étude théorique sur les nouveaux modèles de rôles des patients et médecins, croit que le fait de changer le type de relation de contrôle médecin-patient requiert des changements de comportements du médecin, des clients et clientes, aussi bien que de la structure des lieux de distribution des soins de santé.

Les données que nous avons recueillies sur les contextes de travail aident à comprendre la relation femme violentée-médecin ou infirmière. À l'urgence, la nature et la gravité des problèmes présentés déterminent l'orientation des cas; la spécialisation des fonctions caractérise les tâches des diverses professions; de plus, la procédure de collaboration entre professionnels est établie d'avance et laisse peu de place aux initiatives personnelles (Le Bossé et al., 1989). Nos données sur l'intervention révèlent que les blessures y sont traitées prioritairement, que les médecins recommandent parfois I'hospitalisation pour mieux rejoindre la femme violentée. Le support est difficile à apporter à cause des contraintes de temps et d'énergie. Les suivis ne sont presque pas possibles. Une infirmière insiste d'ailleurs pour dire que le rôle de l'urgence en est plutôt un de dépistage; une autre infirmière réclame plus de sensibilisation à ce propos étant donné le grand nombre de femmes violentées qui s'y présentent.

L'unité de clinique externe se caractérise par le travail d'équipe, les réunions hebdomadaires des médecins, la collaboration de même que la diversité des tâches (soins, enseignement, recherche) (Le Bossé et al., 1989). Il est intéressant de noter chez nos sujets l'intérêt envers les sentiments pouvant accompagner la démarche de recherche d'aide, telle la peur du conjoint. Ceci indique une préoccupation pour la personne par delà les blessures physiques. Les fonctions à I'unité de clinique externe laissent plus de disponibilité pour revoir les femmes violentées lors d'un suivi ou d'une thérapie. Le " désir d'engager la femme violentée ", " de ne pas faire le travail à sa place » et de la "supporter dans l'acquisition de sa liberté » sont des ingrédients de la relation de travail que nous avons recensés.

Quant aux médecins de pratique privée, ils ou elles travaillent de façon totalement indépendante, se regroupent " en cellule -» et ont peu d'occasions de travailler avec d'autres intervenants et intervenantes. Parfois, une clinique peut assurer la formation continue (Le Bossé et al., 1989). Le relevé des actions de ces médecins tant auprès des femmes violentées qu'auprès du milieu reflète un fonctionnement 
reposant sur l'initiative individuelle. Si, d'une part, certains médecins sont favorables à l'idée d'assurer un suivi et une thérapie, à exprimer leur solidarité, à utiliser une approche psychodynamique à base du transfert, d'autres se disent "démunis " après le constat de la violence et n'avoir que le pouvoir de constatation devant ce problème (le critère dépistage est pourtant très important). La collaboration que certains préconisent avec les intervenants et intervenantes du milieu ne paraît pas leur aider à maintenir la relation avec la femme violentée, ni ne consister en une réelle coopération (Johnson, 1983). Enfin, si l'examen de quelques conditions organisationnelles incite à croire que la rencontre intervenant ou intervenante - femme violentée puisse être influencée par le contexte de travail, ce n'est pas là la seule influence. Il faut tenir compte aussi de l'éthique professionnelle de chaque intervenant dans la mesure où nous croyons que ses valeurs et ses attitudes entrent en jeu dans la relation établie. Le dilemme se situera entre ces diverses attitudes : imposer ses valeurs, en faire part ou ne pas en faire part.

\section{L'éthique professionnelle}

Parmi les comportements d'aide recensés, il en est deux qui concernent particulièrement l'éthique professionnelle : « contribuer au scénario de protection " et "divulguer sa position contre la violence ".

Un aspect du scénario de protection, soit celui de recommander le départ du foyer, a été l'objet de bien des travaux (Pagelow, 1984; Rounsaville, 1979). D'un côté, l'Ontario Medical Association (OMA, 1986) met les professionnels et professionnelles en garde contre le fait d'indiquer, par cette recommandation, à la femme violentée, qu'on ne reconnaît pas sa capacité à prendre sa vie en main, qu'on la juge, qu'on la victimise à nouveau. En d'autres mots, on suggère de respecter ses besoins. D'un autre côté, une étude théorique portant sur quelques principes de l'éthique biomédicale, dont la bienfaisance ("beneficience »), rend compatible à l'éthique professionnelle le fait de suggérer à une femme violentée de quitter le foyer (Huston, 1984). Outrepasser l'autodétermination de la cliente se fait dans le but d'assurer sa protection. Le point crucial de l'argumentation s'appuie sur le syndrome de la femme battue immobilisée par la peur, par la dépression et difficilement compétente pour prendre seule une telle décision (Huston, 1984). Le syndrome de la femme battue peut aussi être retracé chez nos sujets : les femmes violentées ne "pensent pas grand-chose d'elles-mêmes", "elles sont dévalorisées", "sont seules ", " ont peur ", " n'ont personne à qui se confier ". Les sujets de notre recherche voient dans cette recommandation une mesure de protection, en laissent l'initiative à la femme violentée après avoir 
exprimé leur opinion et, parfois, mettent des conditions à ce départ. Ainsi on recommande que la femme violentée " prenne le temps de mûrir le projet ", "qu'elle soit prête à quitter. "

Pour souligner la complexité d'une telle recommandation, il est intéressant de la mettre en relation avec l'attitude sous-jacente à la plupart des types d'intervention repérés consistant à transmettre aux femmes qu'elles ont la responsabilité de se prendre en main afin de réagir à la violence conjugale (Le Bossé et al., 1989). Cette attitude est particulièrement perceptible dans la tendance de nos sujets à ne pas prescrire de médicaments pour le problème de violence comme tel et à demander à la femme violentée de faire un choix autre que de subir cette situation-là, dans l'incitation à réagir à sa position contre la violence et à saisir les liens entre la violence et sa santé, et enfin dans la recommandation de porter plainte.

Divulguer sa position contre la violence conjugale relevait, il n'y a pas si longtemps, de convictions personnelles. Maintenant qu'un slogan comme "la violence, c'est inacceptable " est sur la place publique, nous pourrions nous attendre à ce que la position des intervenants et intervenantes puisse être connue des femmes violentées. Garder le silence sur sa position peut être interprété comme acquiescer au mythe de la responsabilité de la femme et à l'absence d'espoir de sortir de la situation. II est suggéré plutôt d'exprimer verbalement ses valeurs sur la situation de la femme violentée incluant un plan pour leur changement (Huston, 1984). En conclusion de leur étude portant sur la réponse à la recherche d'aide de cent neuf femmes violentées, Dobash, Dobash et Cavanagh (1985) constatent que plusieurs médecins adhèrent encore à des mythes concernant la violence faite aux femmes. Les auteures incitent les médecins à adopter un rôle "d'advocacy " et à procurer un support émotionnel et une assistance matérielle tout en exprimant clairement une opposition à la violence et à la domination masculine.

Nos sujets ont été amenés à s'exprimer de façon assez spontanée concernant cette position. La désirabilité sociale a pu les inciter à donner une réponse positive à notre question sur ce point. L'élaboration de leur réponse les a remis en situation d'entrevue avec une femme violentée : on lui exprime que la violence comme telle est inacceptable dans la société et, en même temps, et la prenant à partie, qu'elle n'a pas à subir cette violence, ni d'ailleurs son enfant, à cause de sa valeur personnelle, de ses droits et de nos choix collectifs.

\section{La contribution au système d'aide}

La nécessité de synchroniser la contribution des intervenants et intervenantes de diverses professions apparaît évidente à la lecture de nos données, illustrant l'importance de l'aide ponctuelle à apporter à 
la femme violentée arrivant en situation de crise et la difficulté de poursuivre en milieu médical le travail sur les aspects psychosociaux du problème.

Jetons d'abord un regard sur la préparation que nécessite l'intervention. Le profil de nos sujets indique qu'une infirmière (sur les cinq interviewées) a reçu une formation spécifique sur la violence conjugale alors qu'une autre a reçu des informations juridiques sur ce sujet. Quant aux médecins, aucune formation spécifique sur cette problématique n'est mentionnée. Cependant, une personne du groupe des médecins a déjà été consultante dans une maison d'hébergement pour femmes violentées et mentionne une formation acquise "sur le tas " auprès des pairs, psychologues, travailleuses et travailleurs sociaux. Autre donnée intéressante : plus du quart de nos sujets font référence à la connaissance de la problématique suite à des situations semblables dans leur entourage.

L'intervention en situation de crise ayant fait l'objet de quelques travaux, certains éléments peuvent être utilisés pour comparer l'intervention décodée ici chez nos sujets en milieu médical. On songe aux composantes de l'intervention en situation de crise auprès des femmes violentées, telles que développées par Gilliland et James (1988) comprenant : I'écoute active, le support, l'utilisation du rôle de facilitateur pour que la femme violentée se mobilise pour agir, le fait d'assurer la sécurité de la femme, I'utilisation du rôle "d'advocacy " dans le réseau formel et dans le réseau informel d'aide et enfin prévoir d'autres crises subséquentes. Outre ce dernier aspect, les autres composantes sont présentes chez nos sujets. Des liens peuvent aussi être retracés par rapport à la recherche portant sur l'application du modèle d'intervention de Ginette Larouche et réalisée auprès de travailleuses sociales suivant une formation à cet effet (Pâquet-Deehy et Rinfret-Raynor, 1989). Dans la première phase du modèle d'intervention, I'entrevue reliée au choc de l'agression comprend l'insistance sur le vécu relié à la crise (assurer l'abaissement des tensions émotives et assurer une sécurité physique) et la prise d'une décision initiale (aider la femme battue si elle quitte l'agresseur, I'aider aussi si elle retourne avec l'agresseur). Nos données sur l'offre de support rejoignent l'insistance sur le vécu relié à la crise. L'aide pour la décison initiale est assez explicitée chez nos sujets, mais peu semble fait pour celles qui décident de rester avec le conjoint.

Chez les infirmières, un modèle " $d^{\prime} a d v o c a c y$ " préconise un type d'intervention offrant un support émotionnel, une intervention visant à briser le cycle de violence, le traitement médical, le counseling, l'information sur les services disponibles dans l'hôpital et la communauté (Griffith-Kenney, 1986). Ce modèle s'apparente à nos données sauf que nos sujets n'ont pas utilisé la notion du cycle de violence. 
Au terme de cette discussion des résultats, nous pouvons tenter de préciser le champ de responsabilité des médecins et du personnel infirmier. Sans contredit, le dépistage et l'évaluation de la gravité leur reviennent. Les aspects psychosociaux ont à être reconnus et abordés par eux. La thérapie de couple est contre-indiquée car il s'agit d'abord de se centrer sur les problèmes individuels reliés à la violence avant de travailler sur la relation du couple (Larouche, 1987; Bograd, 1985). $\mathrm{Vu}$ les contraintes reliées au temps, à la disponibilité, à la spécificité de l'intervention médicale, à la non-préparation à l'intervention psychosociale en cette matière, des priorités peuvent être établies dans la liste des comportements d'aide. L'intervention en milieu médical par les infirmières et les médecins peut être reformulée ainsi : l'action auprès des femmes violentées comprendrait : 1) procurer les soins physiques, 2) offrir un support, 3) divulguer sa position contre la violence, 4) contribuer au scénario de protection, 5) s'assurer d'un suivi par un intervenant psychosocial. L'action avec le milieu consisterait à : 1) informer sur l'aide disponible, 2) référer aux ressources communautaires, 3 ) collaborer avec les intervenants et intervenantes du milieu et 4) contribuer aux aspects légaux.

On réalise ici que l'intervention à moyen et à long terme serait confiée à des intervenantes et intervenants psychosociaux, favorisant plutôt pour les intervenants et intervenantes du monde médical une fonction de dépistage systématique, une intervention précise après le choc de l'agression et, enfin, la tâche de faire les références aux ressources du milieu pour poursuivre l'aide aux femmes violentées.

\section{Conclusion}

Plusieurs des comportements d'aide recensés, qu'il s'agisse des actions auprès de la femme violentée elle-même ou auprès du milieu, visent à ce que se termine cette situation de violence. Cependant, le propos n'est pas univoque pour chacun des comportements d'aide, comme nous l'avons noté. Cette lecture dialectique de nos données a permis de faire ressortir le caractère difficile de ce type d'intervention et de suggérer, en bout de piste, une systématisation tenant compte de la réalité en contexte médical.

Les questions soulevées concernant la relation de travail et la déontologie en cette matière sont d'un intérêt commun à tous les intervenants et intervenantes, qu'ils soient en milieu médical ou ailleurs.

II est à souhaiter que la préparation à l'intervention procède par l'introduction de l'étude de la problématique dans la formation générale des médecins et des infirmières, et lors de séminaires en cours d'emploi, auxquels pourraient collaborer des intervenantes et 
intervenants psychosociaux et des militantes des maisons de femmes. La concertation entre personnes aidantes en serait augmentée.

Enfin, il y a urgence à ce qu'une solidarité s'établisse avec les femmes violentées se présentant aux divers services existants. II importe aussi que les analyses générées par nos recherches aient un impact sur le contexte sociopolitique actuel permettant que perdure cette violence faite aux femmes.

\section{Notes}

${ }^{1}$ Cette recherche a été réalisée grâce aux subventions du Conseil québécois de la recherche sociale et du Conseil de recherches en sciences humaines.

${ }^{2}$ Tout au long de l'article, les expressions "monde médical " et " milieu médical " réfèrent aux médecins (hommes et femmes) et aux infirmiers et infirmières.

\section{Références}

BARDIN, L. (1980). L'analyse de contenu. Paris: Presses Universitaires de France.

BilodeAu, A. (1987). La violence conjugale: recherche d'aide des femmes. Québec : Les publications du Québec.

Bograd, M. (1984). " Family Systems Approaches to Wife Battering : A Feminist Critique », American Journal of Orthopsychiatry, vol. 54, $\mathrm{n}^{\circ} 4$ : 558-568.

Brismar, B., B. Bergman, G. Larsson et A. StranberG (1987). "Battered women : a diagnostic and therapeutic dilemna ", Acta Chirurgica Scandinavia, vol. 153, $\mathrm{n}^{\circ} 1: 1-5$.

Christiano, M.R., E. Pollard, B. Sturdevant, G. Benson, C. Perron et T.T. AMATRUDA (1986). "Battered women: a concern for the medical profession ", Connecticut Medecine, vol. 50, n 2 : 99-103.

Commission d'enquête sur les services de santé et les services sociaux (1987). Programme de consultation d'experts, dossier " Femmes", Québec.

COSGWELL, B.E. (1988). "The walking patient and the revolt of the client: Impetus to develop new models of physician-patient roles ", dans S.K. Steinmetz (éd.), Family and support systems across the life span. New York, Londres : Plenum Press.

Dobash, R.E., R.P. Dobash et K. CavanaGh (1985). "The contact between battered women and social and medical agencies", dans J. Pahl (éd.), Private violence and public policy: The needs of battered women and response of the public services. London : Routledge et Kegan.

FINE, M. (1988). The politics of research and activism in studying violence against women. Institut canadien de recherche pour l'avancement des femmes (ICREF).

FORTIN, D. (1988). Le chômage d'insertion: Étude de bénéficiaires d'aide sociale de moins de 30 ans. Thèse de doctorat. Québec : Université Laval.

Ghent, W.R., N.P. DASYlva et M.E. Farren (1985). « Family violence : Guidelines for recognition and management ", Canadian Medical Association Journal, vol. 132, $\mathrm{n}^{\circ} 5$ : 541-553.

Gilliland, G.E. et K. James (1988). "Women in crisis : Battering » (Chap. 4), dans Crisis Intervention Strategies. Pacific Grove, CA: Brooks/Cole Publishing.

GoldberG, N.G. et M.C. Tomlanovich (1984). " Domestic violence victims in the emergency department : New findings ", Jama, vol. 251, n 24 : 3259-3264. 
Gouvernement du Québec (1985). Une politique d'aide aux femmes violentées. Québec, Ministère des affaires sociales.

Griffith-KenNeY, J. (1986). "Abuse and battering " (Chap. 10), dans J. GriffithKenney (éd.), Contemporary women's health: A nursing advocacy approach. Menlo Park : Addison-Wesley.

Huston, K. (1984). "Ethical decisions in treating battered women », Professional psychology : Research and Practice, vol. 15, $\mathrm{n}^{\circ} 6: 822-832$.

Johnson, L.C. (1983). Social Work Practice : A Generalist Approach. Toronto : Allyn and Bacon.

KNIGHT, R.A. et S. HATTY (1987). "Theoretical and methodological perspective in domestic violence : Implications for social action ", Australian Journal of Social Issues, vol. 22, $\mathrm{n}^{\circ} 2: 452-464$.

LALONDE, M.-L. (1976). Modèle de pratique du service social personnel en milieu scolaire. Rapport de recherche. Montréal : Université de Montréal.

LAROUCHE, Ginette (1987). Agir contre la violence. Montréal : Les éditions de la Pleine Lune.

LAVOIE, F. et G. MARTIN (1989). L'intervention des infirmières auprès des femmes violentées par leur conjoint. Article soumis pour publication.

Le Bossé, Y., F. Lavoie et G. Martin (1989a). Attitudes des professionnels-es de la santé vis-à-vis de la violence conjugale. Article soumis pour publication.

Le Bossé, Y., F. LAVOIE et G. MARTIN (1989b). Influence du contexte de travail des professionnels-es de la santé en regard de leurs attitudes vis-à-vis de la violence conjugale. Article soumis pour publication.

L'ÉCUYER, R. (1985). "L'analyse de contenu : notion et étapes ", dans J. Deslauriers (éd.), La recherche qualitative. Chicoutimi : GRIR, Collection Renouveau méthodologique, Université du Québec à Chicoutimi.

LINCOLN, Y. et E. GubA (1985). Naturalistic Inquiry. Beverly Hills, CA : Sage.

MACLEOD, L. (1987). Pour de vraies amours... Prévenir la violence conjugale. Ottawa : Conseil consultatif canadien sur la situation de la femme.

Ontario Medical Association Committee on Wife Assault (1986). Ontario Medical Association Report on Wife Assault (numéro spécial). Ontario Medical Review, décembre : 10-20.

Pagelow, M.D. (1984). Family violence. New York : Praeger.

Pâquet-Deehy, A., M. Rinfret-RAynor et G. Larouche (1989). Apprendre à intervenir auprès des femmes violentées: une perspective féministe. Rapport de recherche. Montréal : Université de Montréal.

PATTON, M.J. (1980). Qualitative evaluation methods. Beverly Hills, CA : Sage.

Rounsaville, B.J. (1978). "Battered wives: Barriers to identification and treatment ", American Journal of Orthopsychiatry, vol. $48: 487-494$.

St-OnGE, M., G. MARTIN et F. LAVOIE (1990). L'évaluation de la violence conjugale par les intervenants-es en milieu médical. Article soumis pour publication.

StARK, E. et A. FLITCRAFT (1988). "Violence among intimates. An epidemiological review » (Chap. 13), dans V. Van Hasselt, R. Morrison et A. Bellach. (éd.), Handbook of Family Violence. New York, London : Plenum Press.

Stark, E., A. Flitcraft et W. Frazier (1979). "Medecine and patriarchal violence : The social construction of a private event ", International Journal of Health Services, vol. 9, $\mathrm{n}^{\circ} 3:$ 461-493.

WARSHAW, C. (1989). "Limitations of the medical model in the care of battered women ", Gender and Society, vol. 3, n 4 : 506-517. 